rheuma plus $2016 \cdot 15: 23$

DOI 10.1007/s12688-016-0075-1

Online publiziert: 3. Juni 2016

(c) Springer-Verlag Wien 2016

CrossMark

\section{Burkhard Leeb}

II. Medizinische Abteilung, NOE Kompetenzzentrum für Rheumatologie, Stockerau, Österreich

\title{
Es wird wieder spannend in der Rheumatologie
}

\section{Liebe Leserinnen und Leser!}

Der Sommer naht und damit auch die zweite Ausgabe von „Rheuma plus“ im Jahr 2016 - wie auch der EULAR 2016 in London.

\section{Erwartungen an den EULAR- Kongress}

Neue Biologika, Biosimilars, aber auch small molecules werden vorwiegendes Thema dieser Veranstaltung sein. Der Ruf nach einer verstärkt individualisierten Behandlung, weg von bloßen Mittelwertanalysen wird lauter werden. Werden Biosimilars es ermöglichen, auch PatientInnen mit niedrigerer Krankheitsaktivität $\mathrm{zu}$ behandeln? Und wird für diese Betroffenen dann das NutzenRisikoverhältnis positiv sein? Es wird wieder spannend in der Rheumatologie und, sollte das Thema Osteoarthritis vermehrt Beachtung finden, dann sogar sehr spannend. Die p.t. Leserschaft wird entscheiden, ob diese Erwartungen an den EULAR in Erfüllung gehen werden; ein möglicher impact des EULAR 2016 könnte die nächsten Ausgaben prägen.

In diesem Zusammenhang besteht universeller Konsens, dass Biologika die konservative Behandlung zahlloser Erkrankungen revolutioniert haben, akute und invalidisierende Krankheitsbilder $\mathrm{zu}$ chronischen Störungen mit relativer Lebensqualität haben werden lassen und damit aus den Behandlungsplänen immunologisch bedingter Störungen nicht mehr wegzudenken sind. Auch von den in der Rheumatologie auf den Markt drängenden small molecules ist Ähnliches zu erwarten.

\section{Immunsuppression und Infektionsrisiko}

Ihnen allen ist die immunsupprimierende oder-modulierende Wirkung gemeinsam und je nachdem, an welcher Stelle und in welchem Ausmaß dies geschieht, muss mit dem Auftreten von Infektionen als Nebenwirkung gerechnet werden. Gleichzeitig senkt eine erfolgreiche Therapie das krankheitsbedingte Grundrisiko, sodass immer ein individueller Nettoeffekt ermittelt werden kann. Florian Thalhammer widmet sich diesem Thema aus infektiologischer Sicht und liefert damit einen Beitrag zur weiteren Individualisierung der Therapie.

\section{Therapie tumorbedingter Schmerzen}

Den Wandel der Schmerztherapie, 30 Jahre nach Einführung des WHOStufenschemas zur Therapie tumorbedingter Schmerzen, beleuchtet Wolfgang Jaksch, der den Fokus auf einen differenzierten Einsatz der Analgetika, nach dem zugrundeliegenden Mechanismus der Schmerzentstehung und dem Ausmaß des Therapieerfolges, legt.

\section{Der Placebo-Effekt in der Schmerztherapie}

In diesem Kontext widmet sich Claus Derra einem zumeist im Hintergrund gehaltenen, darum aber nicht minder wichtigen Phänomen, nämlich dem PlaceboEffekt in der Therapie, mit einer praxisnahen Annäherung: Placeboeffekte nutzen - Noceboeffekte identifizieren und vermeiden. Damit lässt sich vielleicht das Ausnützen dieses Effektes, der, wie man weiß, auch von der Art der Verabreichung des Placebos abhängig ist (je eindrucksvollerverabreicht, umso besser wirksam), erleichtern.

Auch mit dieser Ausgabe hoffen wir wieder dem Ziel von „Rheuma plus“ nahe $\mathrm{zu}$ kommen, eine fruchtbringende und offene Diskussion in Gang zu bringen, aus der wir alle Nutzen ziehen können. Wie immer sage ich für Kritik, Hinweise, Zustimmung und für jeden anderen Beitrag auch schon im Voraus Danke.

Herzlichst,

Ihr Burkhard Leeb

\section{Korrespondenzadresse}

B. Leeb
I. Medizinische Abteilung,
NOE Kompetenzzentrum für
Rheumatologie
Stockerau, Österreich
Burkhard.Leeb@
stockerau.lknoe.at

Interessenkonflikt. B. Leeb gibt an, dass kein Interessenkonflikt besteht. 